\title{
Medios alternativos de Medellín. Tendencia de los temas en su agenda
}

\author{
Alternative Media in Medellín. \\ Trend of issues on its agenda
}

Recibido: 25/06/2020

Aceptado: 01/10/2020

Publicado: 30/11/2020
Mónica Valle Flórez mmvalle@elpoli.edu.co https://orcid.org/0000-0003-1803-1115 Politécnico Colombiano Jaime Isaza Cadavid

Resumen: En este artículo se enuncia la tendencia de los temas que se visibilizan en los medios alternativos de comunicación de Medellín. Los datos corresponden a una revisión aleatoria, no sistemática, de una muestra seleccionada a conveniencia. Es importante destacar que se constituye en la primera observación de dicha agenda, lo que a futuro podría impulsar líneas de investigación al respecto y la creación de un observatorio de estos medios, que posibilite su estudio y permanente apoyo en bien de la construcción de comunicación e información alternativa, popular, ciudadana y comunitaria. Al dirigir la atención hacia la agenda de los medios alternativos, se está alcanzando, quizá un tercer nivel en el análisis de estos, después de su caracterización y expedición de la política pública con la que se pretende el fortalecimiento de las redes de medios, su capacitación, visibilización y acceso de las comunidades a estos.

Es evidente que los medios alternativos son espacios que apoyan (desde lo local y regional) la conciencia ciudadana, los derechos humanos; abordan y problematizan temas de importancia social tales como la drogadicción, la 
inclusión, la tercera edad, la seguridad, el empleo, la organización social, etc. Más allá de la agenda, hay preguntas que enriquecerían estudios de este tipo: ¿quién o quienes direccionan las agendas de los medios alternativos?, ¿las agendas de estos medios coinciden con la de las comunidades en las que se localizan?, ¿la agenda de estos medios tiene algún tipo de escalamiento a otros medios, instancias gubernamentales, acciones de las comunidades, generan opinión pública, cambios, etc.?

Palabras clave: Medios alternativos, Agenda-setting, Comunicación, Comunidad, Opinión pública, Ciudadanía.

Abstract: In this paper, the trend on the issues that are visible in the alternative media in Medellin is stated. Although the data correspond to a random, non-systematic review of a sample selected at the convenience of the author; it is important to point out that it is the first observation of this agenda, which in the future could promote lines of research in this regard, and the creation of an observatory of these media, which allows its study and ongoing support for the construction of. By drawing attention to the alternative media agen$d a$, perhaps a third level is being reached in the analysis of these, after their characterization and construction of a public policy that seeks the creation and strengthening of media networks, training, visibility and access of communities to such media alternatives.

It is evident that alternative media are spaces that support (from the local and regional level) citizen awareness, human rights, address and problematize issues of social importance such as drug addiction, inclusion, old age, security, employment, social organization, etc. Beyond the agenda, there are questions that would enrich studies of this type. Who manage the agendas of the alternative media? Does the agenda of these media coincide with that of the communities in which they are located? Does the agenda of these media have any kind of involvement in other media, governmental instances, community actions, generate public opinion, changes, among others?

Key words: Alternative media, Agenda-setting, Communication, Community, public opinion, Citizenship. 


\section{Introducción}

La agenda-setting es una teoría que plantea que los medios de comunicación difunden noticias y temas que influencias a las audiencias, en el sentido de la percepción de cuáles son los más importantes o destacados del momento.

Los estudios de agenda-setting identifican los temas que componen la agenda mediática, miden la relevancia que en ella se les otorga y la confrontan con la agenda del público para medir el grado de correspondencia entre ambas agendas. La teoría subraya la existencia de una relación causal entre una y otra jerarquización. La repetición, la permanencia en el tiempo, la ubicación de la noticia, la extensión, si se trata de prensa, o la duración en el telenoticiero, son algunos instrumentos que definen la tematización en la agenda mediática. Estudiar la agenda mediática, entonces, permite sopesar la visibilidad de los temas ofertados al público (Sánchez Vilela, 2013).

La introducción del anglicismo "agenda-setting" se atribuye a Donald Shaw y Maxwell McCombs. Estos académicos investigaron el proceso de la agenda pública y la jerarquía de temas que otorgan. Sin embargo, es de advertir que desde 1922 varios investigadores se han ocupado de la agenda de los medios: Walter Lippmann introduce en 1922 la relación entre la agenda de los medios y la agenda de los públicos en su obra Public Opinion. Paul Lazarsfeld y Robert Merton (con fundamento en sus estudios adelantados entre 1948 y 1964) identificaron las funciones de los medios y su papel al momento de destacar los temas. Uno de sus artículos más conocidos de Merton y Lazarsfeld fue La comunicación en masa, el gusto popular, y la acción social organizada: en dicho texto expresan su preocupación por el uso de los medios masivos por parte de los grupos de interés, para ganar control social (Valle Flórez, 2011).

En 1964, Bernard Cohen, introduce la metáfora de la agenda-setting, al indicar que "la prensa no tiene mucho éxito en decir a la gente qué tiene que pensar, pero sí lo tiene al decir a sus lectores sobre qué tienen que pensar" (citado en Rodríguez, 2004, p. 26). Rober Cobb y Charles Elder, entre 1972 y 1983, analizan la agenda política. G. Ray Funkhouser, en 1973, estudia la agenda pública a nivel macro y la relación de esta con la de los medios; 
en 1987, Sahanto Lyengar y Donald Kinder, investigan la agenda pública a nivel macro (Rodríguez, 2004) En la actualidad, los estudios de agenda-setting, se han renovado:

La tendencia de los artículos más recientes indica un alejamiento de la cuestión electoral ... el enfoque de la canalización temática se utiliza actualmente en un amplio abanico de cuestiones: historia, publicidad, información internacional o noticias médicas (Wimmer \& Dominick, 1996, p. 372).

La investigación de la agenda de los medios ha pasado por varias etapas, que se inician con el análisis de contenidos de los medios, pasa a la relación entre la agenda de los medios y la agenda del público; medición de la opinión pública, medición de la "agenda" en términos de temas o asuntos hasta llegar a la "agenda setters", esto es, la de los encargados de la selección de los temas o noticias. Según Iyengar y Kinder (citados en Rodríguez, 2004), "el hecho de que los medios y los gatekeepers o seleccionadores de noticias tengan que decidir qué es publicable y qué no, la forma de hacerlo y el estilo, determina un marco de acción denominado efecto priming o preparación del contexto noticioso que va a percibir la audiencia".

Nuevos estudios se enfocan al análisis de la relación que se establece entre la agenda propuesta por los medios tradicionales y la agenda ofertada por otros grupos de interés o movimientos sociales emergentes que luchan por transformar realidades “políticas, sociales y económicas” (Ríos et al., 2018, p. 97). Las conclusiones de dichas investigaciones han resaltado tres dimensiones: “a) el papel central de las instituciones y las organizaciones en fijar la agenda; b) el proceso comportamental de la agenda y c) el proceso participativo" (Ríos et al., 2018, p. 97).

Otro de los análisis más actualizados es el del "encuadre", que los medios presentan de los temas o noticias: "el framing no se centra en cuáles temas o tópicos son seleccionados para cubrir en los medios de noticias, sino en las formas particulares en que estos están presentados" (Sánchez Vilela, 2013, p. 44). Desde esta perspectiva, las preguntas se sugieren desde el modo en que están construidos los temas y los discursos, así como por los significados que allí se despliegan. Otros enfoques centran el estudio de los "nuevos espacios públicos: el consumo de noticias locales como lugar de encuentro", enten- 
diendo el medio como un espacio creado a partir de prácticas sociales, de interrelaciones, sustentado, creado y recreado a partir de las prácticas comunes y entrelazadas (Cantú, 2015).

En Latinoamérica la referencia a la comunicación alternativa, popular, ciudadana, comunitaria, ha estado vinculada a los movimientos sociales, a la crítica de la sociedad imperante, la educación y también al desarrollo sociocultural de las comunidades. Entre los académicos que generado conocimiento en torno a este tipo de comunicación están Luis Ramiro Beltrán (Bolivia), Rosa María Alfaro (Perú), Alfonso Gumucio Dagron (Bolivia), Amparo Cadavid Bringe (Colombia), Mario Kaplún (Argentina) y Gabriel Kaplún (Uruguay) entre muchos otros que no sólo investigan la comunicación alternativa, sino que la viven e implementan en sus comunidades.

\section{Política pública de medios alternativos en Medellín}

En agosto de 2019, la ciudad de Medellín (Colombia) creó la política pública de medios y procesos de comunicación alternativos, independientes, comunitarios y ciudadanos, mediante el Decreto 1720003875 . Esta tiene por objetivo promover y fortalecer la comunicación ciudadana de forma equitativa, "como expresión de la participación, la cultura ciudadana y la divulgación democrática de opiniones" (Alcaldía de Medellín, 2019).

Aunque Colombia podría considerarse uno de los países más avanzado en términos de la legislación de medios, los derechos de los denominados medios comunitarios han debido conseguirse con firmeza, constancia y paso a paso. La Carta Constitucional de Colombia de 1991, en el Artículo 20, contempla que:

Se garantiza a toda persona la libertad de expresar y difundir su pensamiento y opiniones, la de informar y recibir información veraz e imparcial, y la de fundar medios masivos de comunicación. Estos son libres y tienen responsabilidad social. Se garantiza el derecho a la rectificación en condiciones de equidad. No habrá censura. (Asamblea Constitucional de Colombia, 1991) 
En el 2002, se expidió la Resolución N719, siendo Ministro de Cultura el antioqueño Juan Luis Mejía Arango, actual rector de la Universidad EAFIT, reconocido por su vinculación a la cultura y la educación. Por medio de esta, se declaró a los medios de comunicación ciudadanos y comunitarios como expresión cultural de la nacionalidad colombiana. En tanto se considera que son

espacios de creación cultural desde lo local y regional que contribuyen a la manifestación de imágenes plurales de nación ... espacios de expresión y difusión cultural que inciden implícita y explícitamente en la construcción de la realidad colombiana y sus referentes ... su carácter sobrepone los intereses colectivos sobre los individuales ... son espacios de lo público que inciden en las practicas ciudadanas ... están generando productos y procesos culturales que redundan en el fortalecimiento de la identidad cultural de la región y en la construcción del proyecto de nación ... se han desarrollado en los últimos años cuantitativa y cualitativamente, y han alcanzado una cobertura nacional con la participación de las diferentes comunidades del territorio. (Ministerio de Cultura de Colombia, 2000)

La idea de una política pública para medios alternativos de la ciudad de Medellín se presentó como una de las recomendaciones del Proyecto Estructuración de la Red de Medios Alternativos para la Convivencia Ciudadana (2006). Dicha red se pensó como estrategia para promover redes de conversación, intercambios de mundos simbólicos y expresivos que ayudaran a construir un nosotros de ciudad. Este estudio fue encargado por la Alcaldía de la ciudad con recursos del BID, a la Caja de Compensación Comfama. A esa fecha, la ciudad contaba con 137 medios alternativos. 


\section{Figura 1}

Presencia de medios alternativos por comuna de Medellín - 2006

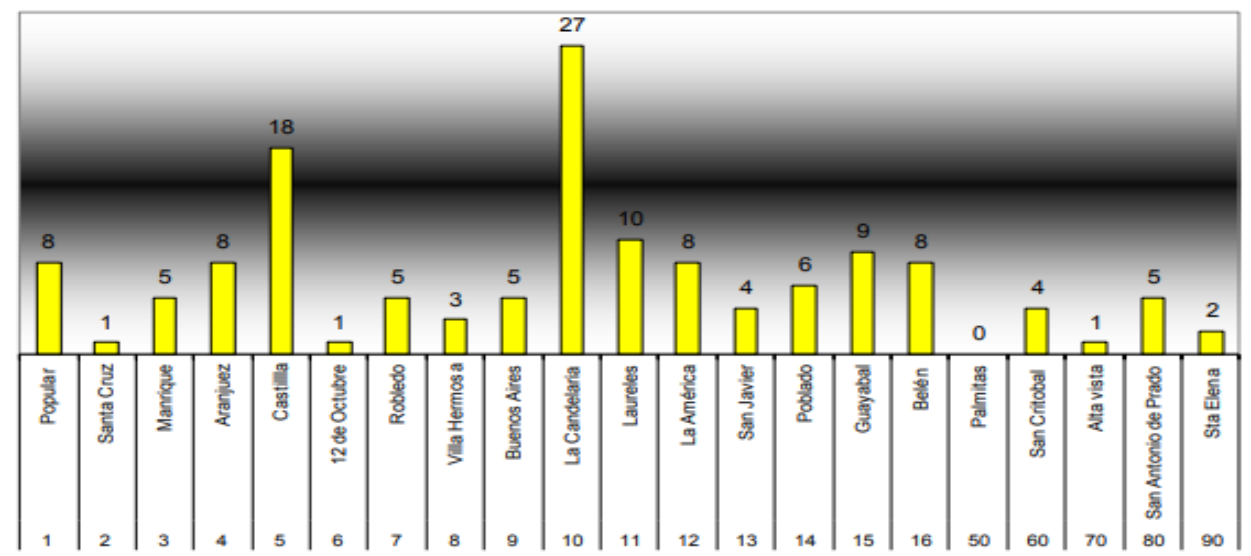

Nota: Valle Flórez (2012a).

En dicho diagnóstico se encontró que el surgimiento de los medios alternativos, era propicio en los sectores que contaban con desarrollo comercial, presencia de organizaciones socioculturales y en aquellos territorios en los que dichos medios eran apoyados con presupuesto participativo, esto es localidades en las que las comunidades acordaban destinar recursos públicos a este ítem. ${ }^{1}$ Desde finales de los noventas, una serie de organizaciones sociales se dieron a la labor de impulsar medios alternativos en esta ciudad, mediante talleres y capacitaciones.

Ese estudio evidenció que los medios alternativos de esta ciudad más que representados buscaban ser reconocidos en sus diferencias. También que se requería impulsar la creación de medios alternativos en las comunas y zonas rurales más desprovistas de estos y fomentar medios alternativos dirigidos a niños (Valle, 2012a).

Otro estudio pionero en la región antioqueña, en torno a los medios alternativos, fue el denominado "Comunicación, Vida y Territorio" CONVITE, realizado por la Gobernación de Antioquia en el año 2005. Este proyecto fue liderado por el Instituto para el Desarrollo de Antioquia (IDEA), con el apoyo

1. Según informe de la Alcaldía de Medellín (2005), el recurso asignado para estos proyectos fue de 519 millones de pesos colombianos. 
de la Universidad de Antioquia y la Caja de Compensación Comfenalco, con el objetivo de fortalecer las capacidades conceptuales, administrativas, técnicas y de cooperación de los medios.

En este estudio se encontró que la región contaba con 308 medios, divididos en 134 emisoras, 120 canales de televisión y 54 impresos. La distribución de los medios por subregiones se presentó así: 20 medios en Urabá, 18 en el Bajo Cauca, 20 en el Nordeste, 28 en el Norte, 27 en el Occidente, 17 en el Magdalena Medio, 53 en el Valle de Aburrá, 80 en el Oriente y 45 en el Suroeste.

\section{Figura 2}

Medios alternativos en Antioquia, Colombia - 2005

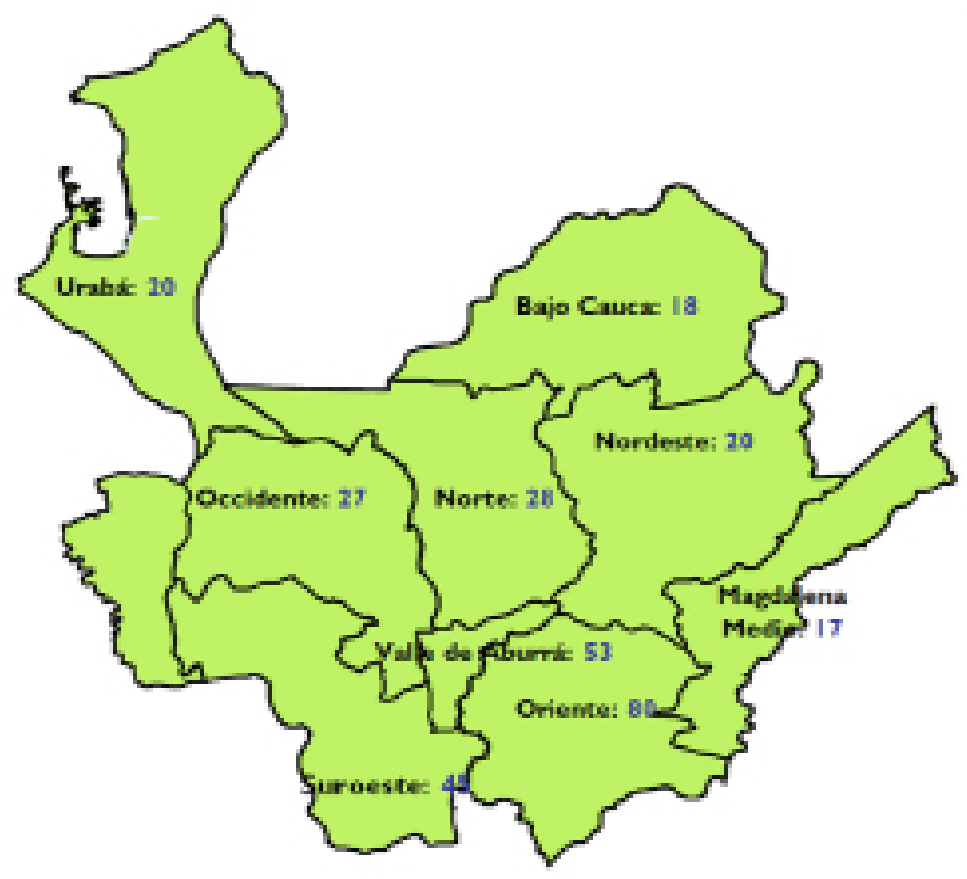

Nota: Valle Flórez (2012b).

El interés por los medios comunitarios llegó a Bogotá, es así como en noviembre de 2005, la Alcaldía Mayor realizó el "Encuentro Distrital de Comunicación Comunitaria", cuyo resultado fue el "Documento de Propuestas de Lineamientos de Políticas en Comunicación Comunitaria para Bogotá” en el que se planteó, entre otros aspectos, la 
inclusión de los medios locales, comunitarios, centros de producción radial comunitarios y colectivos de la comunicación, en la contratación para la divulgación de las campañas comunicativas de promoción de proyectos y programas instituciones del distrito, teniendo en cuenta el potencial de acción de estos medios en términos de su acceso a audiencias locales específicas. (Mesa Bogotana de Comunicación, 2007)

En el contexto capitalino de Bogotá, las comunidades de la ciudad comprenden una pluralidad de escenarios alternativos y comunitarios en el ámbito mediático. Según la Alcaldía Mayor de Bogotá y la Secretaria de Gobierno, en el 2011, en ese territorio se contaba con de 240 medios comunitarios. Estos espacios, clasificados por lógicas de producción (radio, prensa, televisión, TIC), hacen uso de las herramientas periodísticas para incentivar una comunicación participativa, mutable, en constante cambio e hibridación, la cual "no puede ser fácilmente definida porque no puede considerarse un modelo unificado de comunicación" (Chacón, 2017).

En el 2011, conjuntamente con estudiantes del Politécnico Colombiano Jaime Isaza Cadavid, se organizó la primera exhibición de medios alternativos, evento que contó, con dos versiones más en el año 2012. En el 2013, se expide el Acuerdo 73 en el cual se dicta los lineamientos para la creación de la política pública de estos medios

dirigida a promover y fortalecer los procesos comunitarios de comunicación alternativos, independientes, comunitarios y ciudadanos como expresión de la participación, la cultura ciudadana y la divulgación democrática de opiniones. (Alcaldía de Medellín, 2013)

Los principios que inspiraron dicha política fueron el fomento al derecho de la información, la libre expresión, participación ciudadana, identificación, inclusión, articulación, autonomía, coordinación, corresponsabilidad, seguimiento y evaluación (Alcaldía de Medellín, 2013). En el año 2015, la Alcaldía encomienda a la Universidad de Medellín la realización de otro diagnóstico de medios alternativos (Alcaldía de Medellín, 2015). Durante el gobierno de ese año, se adelantaron algunos procesos en torno a la formulación de la Política, pero es en el Plan de Desarrollo Municipal "Medellín Cuenta con vos" (2016-2019) que se asumió el reto "Medellín Participativa" y se creó el proyecto denominado "Medios ciudadanos para la interacción y movilización 
social" con el fin de formular e implementar la política para estos. Se resalta, además, que se pretende proteger la identidad y la memoria de dichos medios.

A partir de ese momento se retoma la mesa articuladora de la política, la cual quedó integrada por medios, gremios y asociaciones del sector de la comunicación además de Empresas Públicas de Medellín (EPM), el Politécnico Colombiano Jaime Isaza Cadavid y las Universidades de Antioquia, UNAD, Luis Amigó, Uniminuto y Pontificia Bolivariana. El Sexto Encuentro de Periodismo Comunitario, que tuvo lugar entre el 16 y 17 de noviembre de 2016, se enmarcó en el proceso de construcción de dicha política. Se realizaron recorridos por la Ciudad para conocer la experiencia de los medios ubicados en las diferentes comunas de Medellín.

Para la visibilización de estos medios, el canal local TeleMedellín, a través del programa "Mi Barrio Cuenta", vinculó a algunos medios alternativos en la producción de material audiovisual, realizó un seriado sobre estos y los capacitó en producción, edición y realización audiovisual (Periódico Viviendo Santa Elena, 2017). A su vez, el Politécnico Colombiano Jaime Isaza Cadavid produjo el documental "Desde la comuna se construye la ciudad" (Revista Luciérnaga - Comunicación, 2018).

La construcción de la política pública de estos medios también pasó por el debate de género. En foro realizado, se abordaron temáticas acerca de la mujer como sujeto de noticia, la importancia de la perspectiva de género y la necesidad de equipos de trabajos mixtos en los medios comunitarios.

Para la identificación y reconocimiento de las dinámicas adelantadas en el proceso de construcción de la política, se entendió la "comunicación popular", como la acción de los movimientos y organizaciones populares que implementan y desarrollan sus propios canales. Dependiendo del tipo de práctica social que la orienta. A esta también se le denomina "alternativa", "participativa", "horizontal”, "comunitaria” y "dialógica". La "comunicación pública" se asumió como aquella que genera procesos de movilización social para el reconocimiento de problemas y la superación de estos mediante una agenda común. El objetivo de este tipo de comunicación es construir contenidos, imaginarios, propósitos realizables y pertinentes para los diversos actores sociales, conservando el respecto a su autonomía e independencia cultural, ideológica o política. 
Los medios alternativos no se constituyen exclusivamente en emisores de mensajes, sino en herramienta importante para la generación de tejido social. Desde esta perspectiva son identificados como ideales, dentro de las nuevas formas de socialización, mientras estén apoyados por la acción de grupos naturales como son las comunidades en donde actúan. Los líderes de los medios comunitarios de Medellín, expresan que su misión es "formar, informar, inconformar y transformar" (Valle, 2012a, p. 44). En estos (y a través de estos), las campañas cívicas encuentran las condiciones necesarias para la difusión de mensajes y la construcción de ciudad.

Lopera (2019) advierte que los audiovisuales realizados por estos colectivos son el resultado de procesos investigativos y metodológicos realizados en amalgama con la comunidad. Esos "audiovisuales Comunitarios" (AC), "documentales sociales participativos" (DSP) y "documentales etnográficos" (DE) contienen la memoria y vivencias de las comunidades. Asimismo, inciden en la identidad y construcción del tejido social comunitario. "Comunicación otra, aquella del buen vivir, el cambio social que impulsa a expresarse y al diálogo de saberes (p. 70).

Desde un ámbito latinoamericano y mundial, se pueden establecer múltiples trabajos investigativos frente a la comunicación alternativa y comunitaria, donde destacan

las reflexiones sobre las dinámicas de los medios alternativos en cuanto a su producción y su acercamiento al medio ciudadano ... el análisis alrededor de la radio indígena ... los estudios de caso de los periodistas en los ámbitos alternativos y comunitarios en las plataformas web ... concepciones y distinciones de comunicación popular, alternativa y comunitaria. (Chacón, 2017, pp. 64-65)

Cuando la política pública de medios alternativos, independientes, comunitarios y ciudadanos de Medellín es un hecho, se ha iniciado la realización del plan de acción, el cual se espera esté terminado a mediados del año 2021. En este contexto, se adelantó el artículo que aquí se presenta y para el que se estableció la siguiente metodología. 


\section{Metodología}

Para evidenciar una posible tendencia de temas propuestos por los medios alternativos de comunicación de Medellín, se dispuso de una muestra a conveniencia de 8 medios impresos. Estos fueron: El Nuevo Sol (Comuna 11), Periódico La Uno (Comuna 1), Epicentro (Comuna 10), Centrópolis (Comuna 10), Contá-Contá (Comuna 13), Presencia 15 (Comuna 15), Signos desde la 13 (Comuna 13) y Universo Centro (Comuna 10). Se advierte que esta muestra no es representativa, por lo que se hace alusión a una tendencia de temas. El estudio se inició con la caracterización de cada uno de los medios que harían parte del corpus a analizar. Se muestra un ejemplo:

Nombre del medio: Presencia 15. El periódico de Guayabal. Distribución gratuita. 20 mil ejemplares. Octubre-noviembre de 2016, año II, número 59. ISSN 2145-7522. Publicación de Corser - Corporación para el ser. Comité editorial N59: JAC Trinidad, Campo Amor y Veeduría C15, Mayorca; Guayabal; Colinita. Sector deporte, Corp. Real juventudes; Sector educativo, Sector ambiental, Mesa ambiental; Colectivo de Mujeres; Comité de inclusión; Sector infancia y juventud, Fundación PJE, Sector social habitantes de la calle; Fundación La Nave de los Sueños; Promotora local de Gobierno, enlace de la Secretaria de la Juventud. Interventoría. Secretaria de Comunicaciones Alcaldía de Medellín.

Se estableció el equipo editorial y de redactores de cada medio. Se muestra un ejemplo:

Medio alternativo: Presencia 15. El periódico de Guayabal. Redactores: Santiago Naranjo M., Lesli Johana Moreno M, Juan Carlos Ramírez M, Alejandro Patiño, Luz Amparo Cardona G. Colaboradores: Colegio San Gabriel de la D. Comunicador Zoológico Santa Fe; Parque Biblioteca MMV; Comunicaciones Inder, Canal Z6; Fundación La Nave de los Sueños. Diseño: Grupo Ingenio. Dirección y edición: Luz Amparo Cardona G.

Se diseñó una plantilla, de la que emergió la información para este estudio, indicándose que los temas (establecidos en los resultados) obedecen al análisis interpretativo hermenéutico realizado y no a las secciones nombradas en los medios, las que en ocasiones no corresponden con el contenido propuesto. De 
todas las posibles correlaciones que se pudieran generar a partir de la información consignada, sólo se expone en este artículo lo relacionado a los temas ( $\sin$ indagar por el encuadre, los emisores, titulares, entre otros), lo que seguramente será objeto de un artículo ulterior en el que se amplíe este estudio.

Tabla 1

Plantilla de sistematización de la información

\begin{tabular}{|c|c|c|c|c|c|c|}
\hline NARRATIVA & SECCIONES & TITULARES & CONTENIDO & $\begin{array}{l}\text { AUTOR I } \\
\text { ILUSTRA- } \\
\text { DOR }\end{array}$ & OBSERVABLE & PUBLICIDAD \\
\hline Opinión & "Editorial" & $\begin{array}{l}\text { Nueva- } \\
\text { mente } \\
\text { en sus } \\
\text { manos }\end{array}$ & $\begin{array}{l}\text { Después de } 10 \text { meses de } \\
\text { obligado receso estamos } \\
\text { nuevamente en sus manos, } \\
\text { para llevar información de } \\
\text { la Comuna } 15 \text { - Guayabal, } \\
\text { el territorio que habitamos. } \\
\text { Convenio con la Alcaldía } \\
\text { N4600066224 de } 2016 \text {. } \\
\text { Coser aporta el } 43 \% \text {. }\end{array}$ & & & $\begin{array}{l}\text { Este medio es } \\
\text { apoyado par- } \\
\text { cialmente con } \\
\text { dineros públicos } \\
\text { priorizados } \\
\text { por habitantes } \\
\text { de la Comuna } \\
\text { 15- Guayabal en } \\
\text { el Programa de } \\
\text { Planeación Local } \\
\text { y Presupuesto } \\
\text { Participativo de } \\
\text { la Alcaldía de } \\
\text { Medellín. }\end{array}$ \\
\hline Perfil & "Gente" & $\begin{array}{l}\text { Jhon Fredy } \\
\text { Moncada } \\
\text { Rojas, un } \\
\text { deportista } \\
\text { sin límites. }\end{array}$ & $\begin{array}{l}\text { La discapacidad es una } \\
\text { situación que puede causar } \\
\text { cambios importantes en } \\
\text { la vida de quien la posee, } \\
\text { pero jamás la anulación de } \\
\text { su vida social y productiva. } \\
\text { Nato, vive en el centro de } \\
\text { la Comuna, en el barrio } \\
\text { Cristo Rey. }\end{array}$ & $\begin{array}{l}\text { Juan Carlos } \\
\text { Ramírez } \\
\text { Monsalve }\end{array}$ & $\begin{array}{l}\text { Refieren a la } \\
\text { constitución } \\
\text { Política de } \\
\text { 1991. Titulo } 1 \\
\text { de los prin- } \\
\text { cipios fun- } \\
\text { damentales. } \\
\text { Artículo 1. }\end{array}$ & \\
\hline Noticia & "Somos" & $\begin{array}{l}\text { Entre } \\
\text { Costuras. }\end{array}$ & $\begin{array}{l}\text { El costurero que busca di- } \\
\text { sipar la cotidianidad y fo- } \\
\text { mentar en sus integrantes } \\
\text { un arte del cual se puedan } \\
\text { lucrar económicamente. } \\
\text { El Telecentro de Trinidad } \\
\text { lleva algún tiempo con- } \\
\text { vocando personas que se } \\
\text { encuentran interesadas en } \\
\text { las manualidades. }\end{array}$ & & $\begin{array}{l}\text { Fotografías de } \\
\text { las integrantes } \\
\text { del costurero }\end{array}$ & Confiar \\
\hline Noticia & "Ambiental" & $\begin{array}{l}\text { Red de } \\
\text { huertas }\end{array}$ & $\begin{array}{l}\text { Una red de huertas, en la } \\
\text { idea de integración que ha } \\
\text { calado entre los impulsores } \\
\text { de huertas y ambientalistas } \\
\text { de la Comuna 15, cada vez } \\
\text { se concreta más para bene- } \\
\text { ficio de todos. Líderes de } \\
\text { huertas como Aldea Verde, } \\
\text { la huerta del Plato, Doña } \\
\text { Pacha, generaron una red } \\
\text { solidaria para compartir } \\
\text { conocimiento.... }\end{array}$ & $\begin{array}{l}\text { Luz A. } \\
\text { Cardona } \\
\text { Galindo }\end{array}$ & $\begin{array}{l}\text { Fotos de las } \\
\text { personas } \\
\text { trabajando en } \\
\text { las huertas }\end{array}$ & \\
\hline
\end{tabular}




\begin{tabular}{|c|c|c|c|c|c|c|}
\hline Crónica & "Inclusión" & $\begin{array}{l}\text { Habitantes } \\
\text { de la calle }\end{array}$ & $\begin{array}{l}\text { Las calles de Guayabal } \\
\text { están llenas de una doble } \\
\text { vida. La primera, aceptable } \\
\text { y ajustada con las normas } \\
\text { de urbanidad y la segunda, } \\
\text { nostálgica, menos lúcida } \\
\text { pero llena de seres huma- } \\
\text { nos igual de valiosos. }\end{array}$ & $\begin{array}{l}\text { Juan Carlos } \\
\text { Ramírez } \\
\text { Monsalve }\end{array}$ & Fotografía & \\
\hline Noticia & "Obras" & $\begin{array}{l}\text { Betania: un } \\
\text { futuro a la } \\
\text { deriva }\end{array}$ & $\begin{array}{l}\text { La obra de ampliación vial } \\
\text { que inició en el sur de la } \\
\text { Comuna 15, beneficiará a } \\
\text { los barrios San Rafael, La } \\
\text { Colina y Betania, en este } \\
\text { última se evidenciará a } \\
\text { comienzos del 2017, con } \\
\text { incertidumbre para algu- } \\
\text { nos habitantes. }\end{array}$ & $\begin{array}{l}\text { Santiago } \\
\text { Naranjo } \\
\text { Mesa }\end{array}$ & Fotografía & \\
\hline Crónica & $\begin{array}{l}\text { "Cosas que } \\
\text { hablan" }\end{array}$ & $\begin{array}{l}\text { Casa Museo } \\
\text { Santa Fe }\end{array}$ & $\begin{array}{l}\text { Hoy la hacienda Santa } \\
\text { Fe, como se le llamaba, se } \\
\text { ha convertido en varios } \\
\text { barrios y la vivienda en } \\
\text { Casa Museo Santa Fe. Este } \\
\text { es un lugar sosegado para } \\
\text { descansar y admirar en } \\
\text { medio del recorrido por } \\
\text { las especies que habitan el } \\
\text { Parque Zoológico donde } \\
\text { está ubicada. }\end{array}$ & $\begin{array}{l}\text { José Ber- } \\
\text { nardo Luna } \\
\text { (Comuni- } \\
\text { caciones } \\
\text { Zoológico } \\
\text { Santa Fe) }\end{array}$ & $\begin{array}{l}\text { Páginas } \\
\text { centrales }\end{array}$ & \\
\hline Entrevista & "Participación" & $\begin{array}{l}\text { Balance } \\
\text { Positivo } \\
\text { para PP y } \\
\text { PL, para } \\
\text { Guayabal / } \\
\text { Planeación } \\
\text { local y } \\
\text { Presupuesto } \\
\text { participa- } \\
\text { tivo. }\end{array}$ & $\begin{array}{l}\text { Seis mil } 911 \text { millones } 378 \\
\text { mil pesos, es el recurso } \\
\text { asignado y que se ejecutará } \\
\text { en } 2017 \text {, en } 16 \text { proyectos, } \\
\text { en la Ruta de PP y PL para } \\
\text { la Comuna } 15 \text { Guayabal. } \\
\text { Entrevista a Juliana Co- } \\
\text { lorado Duque. Técnica } \\
\text { delegada de PL y PP }\end{array}$ & $\begin{array}{l}\text { Juan Carlos } \\
\text { Ramírez } \\
\text { Monsalve/ } \\
\text { Luz A. } \\
\text { Cardona } \\
\text { Galindo }\end{array}$ & & $\begin{array}{l}\text { Charla ¿Quiere } \\
\text { su propio nego- } \\
\text { cio? La mejor op- } \\
\text { ción para generar } \\
\text { ingresos legali- } \\
\text { dad y trabajo en } \\
\text { equipo. Opor- } \\
\text { tunidad única } \\
\text { de economía } \\
\text { participativa. }\end{array}$ \\
\hline Campaña & & $\begin{array}{l}\text { Campaña } \\
\text { sobre cua- } \\
\text { drante de } \\
\text { seguridad }\end{array}$ & & & & $\begin{array}{l}\text { Publicidad Pre- } \\
\text { sencia 15. ¿Tiene } \\
\text { negocio, empresa } \\
\text { o un anuncio? } \\
\text { ¡Llámenos! Pre- } \\
\text { sencia15periodi- } \\
\text { co@gmail.com }\end{array}$ \\
\hline Noticia & "Educación" & $\begin{array}{l}\text { Oportu- } \\
\text { nidad de } \\
\text { educación } \\
\text { superior. }\end{array}$ & $\begin{array}{l}\text { Convocatoria abierta hasta } \\
\text { el } 15 \text { de noviembre del } \\
\text { presente año, en el Fondo } \\
\text { camino a la educación } \\
\text { superior. La Comuna 15- } \\
\text { Guayabal, será parte de } \\
\text { un nuevo proyecto para } \\
\text { fomentar el estudio en } \\
\text { los jóvenes recientemente } \\
\text { graduados en } 2016 .\end{array}$ & $\begin{array}{l}\text { Alejandro } \\
\text { Patiño } \\
\text { Vega }\end{array}$ & & $\begin{array}{l}\text { Fundación PJR. } \\
\text { Investigaciones. } \\
\text { Inglés y Fútbol } \\
\text { gratis para los } \\
\text { jóvenes y niños. }\end{array}$ \\
\hline
\end{tabular}




\begin{tabular}{|c|c|c|c|c|c|c|}
\hline Opinión & “Opinión” & $\begin{array}{l}\text { ¡No } \\
\text { escandalice- } \\
\text { mos a los } \\
\text { niños y } \\
\text { niñas!, } \\
\text { Es lo Justo }\end{array}$ & $\begin{array}{l}\text { Hace pocos días, en una } \\
\text { de las aulas de clase nos } \\
\text { encontramos con uno de } \\
\text { los dilemas trascendenta- } \\
\text { les en la formación de la } \\
\text { ciudadanía, la concepción } \\
\text { de la familia y el reconoci- } \\
\text { miento de la sexualidad del } \\
\text { individuo. }\end{array}$ & $\begin{array}{l}\text { Sergio A. } \\
\text { Cardona, } \\
\text { Gody. } \\
\text { Coordina- } \\
\text { dor Colegio } \\
\text { San Gabriel } \\
\text { de la Dolo- } \\
\text { rosa. }\end{array}$ & $\begin{array}{l}\text { Fotos. Con } \\
\text { efectos para } \\
\text { mostrar a los } \\
\text { niños. }\end{array}$ & \\
\hline $\begin{array}{l}\text { Crónica } \\
\text { noticiosa }\end{array}$ & "Cultura" & $\begin{array}{l}\text { Se aclaran } \\
\text { dudas sobre } \\
\text { espacios de } \\
\text { cultura en } \\
\text { Guayabal }\end{array}$ & $\begin{array}{l}\text { El Parque Biblioteca } \\
\text { Manuel Mejía Vallejo fue } \\
\text { el lugar de encuentro para } \\
\text { hablar de los espacios cul- } \\
\text { turales que debe tener la } \\
\text { Comuna } 15 \text { Guayabal. Los } \\
\text { principales temas fueron: } \\
\text { La segunda etapa de la } \\
\text { construcción del Parque } \\
\text { Biblioteca, el proyecto } \\
\text { Casa de la Música, Casa } \\
\text { de la Cultura y espacios } \\
\text { artísticos. }\end{array}$ & $\begin{array}{l}\text { Juan Carlos } \\
\text { Ramírez } \\
\text { Monsalve }\end{array}$ & $\begin{array}{l}\text { Fotos de la } \\
\text { reunión }\end{array}$ & EPM \\
\hline Noticia & "Deportes" & $\begin{array}{l}\text { Más de } 100 \\
\text { jóvenes } \\
\text { por día en } \\
\text { el Parque } \\
\text { de Ruedas } \\
4 \text { Sur }\end{array}$ & $\begin{array}{l}\text { La Comuna } 15 \text { Guayabal } \\
\text { se ha caracterizado por ser } \\
\text { un lugar de encuentro para } \\
\text { niños, jóvenes y adultos en } \\
\text { el tema de deporte. Ahora } \\
\text { más que nunca se valida } \\
\text { esta información con el es- } \\
\text { cenario deportivo Parque } \\
\text { de Ruedas } 4 \text { Sur. Con una } \\
\text { inversión de } \$ 893 \text { millones } \\
\text { para su construcción y } \\
\text { un área de } 1.800 \text { metros } \\
\text { cuadrados solo destinados } \\
\text { para BMX, Roller y Skate. }\end{array}$ & $\begin{array}{l}\text { Juan Carlos } \\
\text { Ramírez } \\
\text { Monsalve }\end{array}$ & & \\
\hline Noticia & "Salud" & $\begin{array}{l}\text { De roedores } \\
\text { y otros } \\
\text { bichos }\end{array}$ & $\begin{array}{l}\text { Ya es común encontrar } \\
\text { ratas, cucarachas y otros } \\
\text { bichos saliendo por la } \\
\text { alcantarilla de las calles o } \\
\text { en un rincón de la acera } \\
\text { comiendo desperdicios, } \\
\text { Pero tenemos riegos de } \\
\text { enfermedades si no preve- } \\
\text { nimos la propagación. }\end{array}$ & $\begin{array}{l}\text { Luz A. } \\
\text { Cardona } \\
\text { Galindo / } \\
\text { Alejandro } \\
\text { Patiño } \\
\text { Vega }\end{array}$ & $\begin{array}{l}\text { Foto grande } \\
\text { de una rata }\end{array}$ & \\
\hline
\end{tabular}

Nota: Elaboración propia.

\section{Hallazgos}

En la agenda de los medios alternativos de Medellín, se evidencian temas sociales en los que se abordan problemáticas tales como la drogadicción, el empleo informal, espacios para la tercera edad, la importancia de la comunicación, de hablar y escribir correctamente. Esta temática también sirve para resaltar personas como los loteros; en los obituarios, se reseña el fallecimiento de personas de la comunidad. 
¿Qué hacen las organizaciones sociales?, ¿qué organizaciones están liderando dicha agenda? Es necesario procurar que estos medios no se constituyan en los boletines informativos de las instituciones que integran los comités de redacción.

Si bien el tema social es uno de los que más se aborda en los medios de comunicación observados, es necesario indicar que es poca la importancia que ocupa en el medio, en cuanto a espacio, desarrollo de contenido, ubicación y quizá (lo más importante) en cuanto al encuadre. La mayoría de los escritos son de opinión (algunas más ilustradas que otras) y sólo unas pocas de expertos. La pregunta que surge es ¿qué tanto coincide la agenda de los medios alternativos, comunitarios, ciudadanos e independientes con la agenda de las comunidades en las que están insertos?

Figura 3

Temas en la agenda de los medios alternativos de Medellín

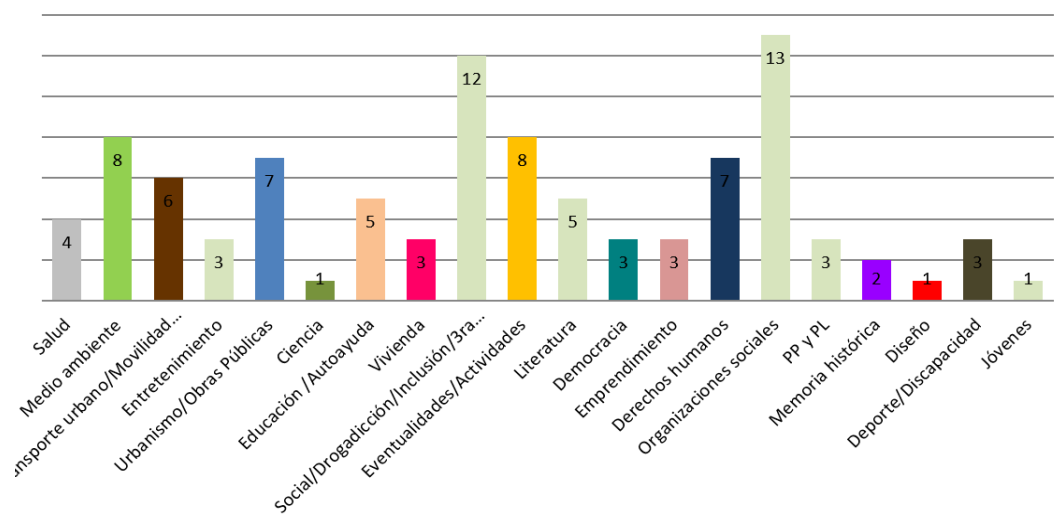

Nota: Elaboración propia.

El tema del medio ambiente ocupa el tercer lugar de visibilidad en los medios alternativos. Entre los subtemas de este ítem identificamos: las ecohuertas, los decretos y normas, la mesa ambiental, el plan integral de gestión de la calidad del aire (PIGECA) y la contaminación. Aunque dicho asunto es totalmente relevante, se hace necesario que los medios se pregunten por el encuadre, tratamiento y desarrollo del tema. El tema ambiental también ocupa la agenda de los medios masivos y de algunas instancias gubernamentales, así que una opinión no especializada al respecto podría considerarse poco importante. Sin duda, la caracterización de las problemáticas medioambientales de 
la localidad podría conllevar a acciones concretas de parte de la comunidad y a un manejo informativo más pertinente.

En espacios como las reseñas, las actividades (eventualidades pasadas o futuras) son otro tema en la agenda de los medios. En tanto la circulación de estos es semestral, bimensual y mensual, es conveniente un tratamiento especial de este tipo de información, para que no pierda vigencia o se termine afectando el nivel de recordación de la actividad. En tal sentido, en la producción del medio se debe tener presente el manejo de los tiempos de la actividad para efectos de redacción, y si esta ya sucedió, está en desarrollo o se realizará a futuro.

El tema de los derechos humanos aparece también en la agenda de los medios consultados, estableciéndose énfasis en la movilización ciudadana, la labor de las mesas de paz y la convivencia; la construcción de procesos sociales, negociaciones de paz, educación para la paz y el posconflicto. El urbanismo y las obras públicas son otros de los temas de interés de estos medios. En estos se informa de asuntos como nuevas vías de acceso al barrio, la ampliación vial, la accidentalidad producida por las motos, la normatividad y campañas de inteligencia vial. El contenido de estas temáticas refleja la preocupación de la comunidad. Asimismo, surge la capacidad de los medios comunitarios por escalar dichas inquietudes a otros medios e instancias gubernamentales. ¿Qué tanta movilización social y escalonamiento de problemáticas se logran desde los medios alternativos?

La literatura se posiciona como un tema relevante en los medios alternativos. Es así que la poesía, los poetas, los cuentos y escritores encuentran un lugar en estas publicaciones. En todo caso, resulta necesario visibilizar mucho más el talento local. El tema educativo se encuadra sustancialmente en perspectivas de la autoayuda, curso y capacitación informal. La salud se enmarca en asuntos como las hierbas medicinales, meditación, la buena alimentación e información acerca de los Comités de Participación Comunitaria en Salud (COPACOS).

La democracia es otro de los temas que se publica en estos medios. El contenido se enfoca especialmente en las votaciones. Sin embargo, se podría dar mayor cubrimiento a asuntos de participación ciudadana, presupuesto participativo y la planeación local. El deporte no es muy relevante en estos medios: lo que llama la atención es que tanto el Instituto de Deportes y Recreación de 
Medellín tiene presencia en muchas de estas comunidades. Más allá de esto, la actividad deportiva es un elemento que aporta a la calidad de vida de las personas y las comunidades.

En estos medios emergen inquietudes relacionados con la vivienda, con temas puntuales tales como las escrituras de los predios, la valorización de los terreros, los derrumbes y la utilización de casa antiguas ("casonas") para usos comerciales. El emprendimiento también está presente en las páginas escritas de los medios alternativos consultados, aunque a estos temas les falta seguimiento, desarrollo y encuadre. Entre las temáticas, que podrían catalogarse como "nuevas", están las referidas a la ciencia y la tecnología, el diseño (en la moda y el cine) y la memoria histórica.

Dentro del espectro del entretenimiento, estos medios publican chistes, sopas de letras, sudoku, crucigramas. Entre las temáticas poco visibilizadas están el sector juvenil, la violencia de género, la seguridad en las comunas, el desempleo, la delincuencia, la violencia intrafamiliar, el abuso infantil, las barreras invisibles, entre otros.

\section{A manera de reflexión}

Visibilizar la tendencia de los temas que se están abordando en los medios alternativos de Medellín permite contemplar un panorama que no se agota, sino que alienta a conocer y estudiar mucho más el fenómeno de los medios alternativos, comunitarios, ciudadanos e independientes. Surgen nuevas preguntas en torno a los medios, que deberán ser respondidas para consolidar y avanzar en el proceso de construcción de comunidades, su derecho a la información y libre expresión: ¿la agenda de las comunidades, la gente del común de un sector, coincide con la agenda de los medios alternativos de cada localidad?, ¿cuál es el problema más importante al que tienen que hacer frente cada comuna o barrio en el que opera el medio alternativo?, ¿qué tanta movilización social se agencia desde los medios alternativos?, ¿quiénes escriben en los medios alternativos?

Al dirigir la atención hacia la agenda de los medios alternativos en Colombia, se está alcanzando un tercer nivel en el análisis de estos, después de su caracterización y la construcción de la política pública, con la que se pretende 
la creación y el fortalecimiento de las redes de medios, la capacitación, la visibilización de estos y su acceso de las comunidades. Es evidente que los medios alternativos son espacios que apoyan (desde lo local y regional) la conciencia ciudadana, los derechos humanos; abordan y problematizan temas de importancia social como la drogadicción, la inclusión, la tercera edad, seguridad, el empleo, la organización social, entre otros. Más allá de la agenda, hay preguntas que enriquecerían posteriores estudios: ¿quién o quienes direccionan las agendas de los medios alternativos?, ¿la agenda de estos medios tiene algún tipo de escalamiento a otros medios, instancias gubernamentales, acciones de las comunidades, generan opinión pública, cambios?

Los medios alternativos deben avanzar en aspectos como el encuadre de las temáticas y su desarrollo; redacción, informes investigativos, denuncias, entre otros. En esencia, los medios deben ser la voz del pueblo y velar por sus intereses. La mayoría de las temáticas están planteadas desde la opinión (algunas desde la opinión técnica y especializada) y sólo algunas están formuladas por expertos. En ese sentido, los medios alternativos deben explorar narrativas como la notica, la crónica, el reportaje, la entrevista y el informe investigativo.

Se evidencia un avance en la diagramación y diseño, así como en la fotografía de estos medios. Del mismo modo, se observa el uso de la ilustración como elemento gráfico. En algunos medios, la definición de la sección o tema no coincide con el contenido, como puede observarse en una sección denominada "Ciudad y el mundo", publicidad de la corporación o medio mismo que lo edita. Se destaca que algunos de los medios cuentan con la sección "La carta del lector" en la que se puede evidenciar algunas de sus preocupaciones del poblador local. Se debe trabajar en la generación de procesos de retroalimentación (o interacción) entre el medio alternativo y su audiencia, lectores y comunidad.

Asimismo, se evidencia una tendencia que permite indicar que la agenda de los medios alternativos está siendo determinada por las organizaciones sociales que integran sus comités editoriales, lo que resulta muy favorable. Sin embargo, estos medios no deben constituirse en boletines informativos de estas instituciones. Las problemáticas enfocadas por dichas instituciones puedan ser desarrolladas, encuadradas e investigadas si son del interés de la comunidad y, por supuesto, visibilizadas a través de estas publicaciones. 
En todos los medios estudiados, se reseña la intervención y acompañamiento de la Secretaria de Comunicaciones Alcaldía de Medellín. Al respecto, surge la pregunta: ¿cuáles han sido los aprendizajes y factores de éxito del acompañamiento que la Alcaldía de Medellín a efectuado a estos medios? Entendiéndose que la agenda de los medios no son la "realidad" y que su influencia en la audiencia no es directa (sino que obedece a varios factores y que hay agendas múltiples y su análisis es altamente complejo) es necesario entender dicho fenómeno, lo cual implica investigación. Al respecto, surgen las interrogantes: ¿coincide la agenda gubernamental con la de estos medios?, ¿cuál debe ser la agenda de estos medios?, ¿quién debe determinar dicha agenda?, ¿cuál ha sido la evolución de las temáticas en estos medios?

En el contenido publicado por los medios observados, no se alcanza a visibilizar los temas relacionados con la violencia de género, la seguridad en las comunas, el desempleo, la delincuencia, la violencia intrafamiliar, el abuso infantil, las barreras invisibles, etc. Los medios alternativos no se constituyen exclusivamente en emisores de mensajes, sino en herramientas importantes para la generación de tejido social. Desde esta perspectiva, son identificados como ideales (dentro de las nuevas formas de socialización) mientras estén apoyados por la acción de grupos naturales como son las comunidades en donde actúan.

\section{Fuentes consultadas}

Alcaldía de Medellín. (2005, Diciembre 28). Boletín 1398 de Comunicaciones.

Alcaldía de Medellín. (2013). Gaceta Oficial N 4207. https://www.medellin. gov.co/irj/go/km/docs/pccdesign/SubportaldelCiudadano_2/Plande-Desarrollo_0_15/Publicaciones/Shared\%20Content/GACETA\%20 OFICIAL/2014/Gaceta\%204207/4207.pdf

Alcaldía de Medellín. (2015). Política pública de medios alternativos, independientes,comunitarios y ciudadanos de Medellín. Diagnóstico de medios. http://media.wix.com/ugd/e86a69_03edf131ca3846adad1445edf4b3d2d6.pdf 
Alcaldía de Medellín. (2019). Decreto 1720003875. Política pública de medios y procesos de comunicación alternativos, independientes, comunitarios y ciudadanos. https://revistas.elpoli.edu.co/index.php/luc/ article/view/Editorial

Asamblea Nacional Constituyente de Colombia. (1991). Constitución Política de Colombia. https://pdba.georgetown.edu/Constitutions/Colombia/colombia91.pdf

Caja de Compensación Familiar de Antioquia, COMFAMA (2006). Proyecto Estructuración de la Red de Medios Alternativos para la Convivencia Ciudadana. https:/es.scribd.com/doc/6919283/ MEDIOS-DE-COMUNICACION-ALTERNATIVOS-PARA-LA-CONVIVENCIA

Cantú, A. (2015). Nuevos espacios públicos: el consumo de noticias locales como lugar de encuentro. Luciérnaga Comunicación, 5(9), 69-77. https://revistas.elpoli.edu.co/index.php/luc/article/view/302

Chacón, J. (2017). Características de los medios comunitarios y alternativos de Bogotá - Colombia. Luciérnaga Comunicación, 8(15), 63-83. https://doi.org/10.33571/revistaluciernaga.v8n15a5

Lopera Rueda, J. (2020). Prácticas, saberes y apropiación del medio audiovisual en los colectivos de comunicación comunitaria de la ciudad de Medellín. Luciérnaga Comunicación, 11(22), 70-83. https://doi. org/10.33571/revistaluciernaga.v11n22a2

Mesa Bogotana de Comunicación. (2007). Documento de Propuestas de Lineamientos de Políticas en Comunicación Comunitaria para Bogotá. http://media.wix.com/ugd/e86a69_25f19e4e7b0045488597f57456586c0c.pdf

Ministerio de Cultura de Colombia. (2000, Junio 7). Resolución No 719. https://xperta.legis.co/visor/temp_legcol_d98cad41-7ba3-46c9-bc25$544453605 \mathrm{ef3}$ 
Periódico Viviendo Santa Elena. (2017, Enero 7). Mi Barrio Cuenta - Capítulo 8 emitido el 28 de diciembre de 2016 [Video]. YouTube. https:// www.youtube.com $/$ watch? $=00 T h X 7 k J V 3 U$

Revista Luciérnaga - Comunicación. (2018, febrero 15). Medios Alternativos de Medellín - El Megáfono [Video]. YouTube. https://www.youtube. $\mathrm{com} /$ watch?time_continue $=8 \& \mathrm{v}=\mathrm{X} 4 \mathrm{z} 8 \mathrm{y} 9 \mathrm{KS} 22 \mathrm{~s} \&$ feature $=\mathrm{emb} \log 0$

Ríos, I., Pérez-Marín, M. y Morillo, S. (2018). La agenda setting en la red social Facebook: Campaña del plebiscito por la paz en Colombia. Kepes, 15(17), 93-117. http://vip.ucaldas.edu.co/kepes/downloads/Revista17_5.pdf

Rodríguez, R. (2004). Teoría de la Agenda-Setting aplicación a la enseñanza universitaria. http://josemramon.com.ar/wp-content/uploads/opinion-publica-evolucion-de-la-teoria-de-la-Agenda-Setting.pdf

Salazar Torres, N. (2017). Comunicación indígena en Colombia. Entre el sentido de lo propio y lo apropiado. Luciérnaga Comunicación, 8(15), 48-62. https://doi.org/10.33571/revistaluciernaga.v8n15a4

Sánchez Vilela, R. (2013). Medios de comunicación y cultura: Frame analysis para el estudio de la recepción. Luciérnaga Comunicación, 5(10). 4154. https:/www.politecnicojic.edu.co/images/downloads/publicaciones/revista-luciernaga/luciernaga-10/pdf/4_frame_analysis.pdf

Valle Flórez, M. (2011). Dimensión de análisis de la investigación en comunicación: hacia el diálogo metodológico. Luciérnaga Comunicación, 3(5), 39-57. https://doi.org/10.33571/revistaluciernaga.v3n5a1

Valle Flórez, M. (2012a). Medios Alternativos de Medellín [1]: Recuento Histórico (1987-2006). Luciérnaga Comunicación, 4(7), 36-46. https://doi.org/10.33571/revistaluciernaga.v4n7a4

Valle Flórez, M. (2012b). Medios Alternativos de Medellín [2]: Diagnóstico 2005-2006. Luciérnaga Comunicación, 4(8), 51-57. https://doi. org/10.33571/revistaluciernaga.v4n8a3 
Valle Flórez, M. (2017). Avanza la política de medios alternativos, independientes, comunitarios y ciudadanos de Medellín. Luciérnaga Comunicación, 8(16), 4-9. https://doi.org/10.33571/revistaluciernaga. v8n16a1

Wimmer, R., \& Dominick, J. (1996). La investigación cientifica de los medios de comunicación. Una introducción a sus métodos. Bosch. 